\title{
A Cancellation Nanoflare Model for Solar Chromospheric and Coronal Heating
}

\author{
E. R. Priest ${ }^{1}$ (i), L. P. Chitta ${ }^{2}$ (i), and P. Syntelis ${ }^{1}$ (i) \\ ${ }^{1}$ School of Mathematics and Statistics, University of St Andrews, St Andrews, Fife KY16 9SS, UK; ps84@ @t-andrews.ac.uk \\ ${ }^{2}$ Max Planck Institute for Solar System Research, D-37077, Göttingen, Germany \\ Received 2018 June 21; revised 2018 July 16; accepted 2018 July 20; published 2018 August 1
}

\begin{abstract}
Nanoflare models for heating the solar corona usually assume magnetic braiding and reconnection as the source of the energy. However, recent observations at record spatial resolution from the SUNRISE balloon mission suggest that photospheric magnetic flux cancellation is much more common than previously realized. We therefore examine the possibility of three-dimensional reconnection driven by flux cancellation as a cause of chromospheric and coronal heating. In particular, we estimate how the heights and amount of energy release produced by flux cancellation depend on flux size, flux cancellation speed, and overlying field strength.
\end{abstract}

Key words: magnetohydrodynamics (MHD) - magnetic reconnection - Sun: atmosphere - Sun: chromosphere Sun: corona - Sun: magnetic fields

\section{Introduction}

Many interesting proposals have been put forward to solve the major puzzle of how the solar atmosphere is heated, including magnetohydrodynamic (MHD) waves and magnetic reconnection (e.g., Klimchuk 2006; Parnell \& De Moortel 2012; Priest 2014). However, the mechanisms have not yet been conclusively identified. The classic picture for nanoflares invokes the magnetic braiding of footpoints to create many current sheets that dissipate by reconnection throughout the corona (Parker 1988). This was later developed into the coronal tectonics model (Priest et al. 2002), with dissipation at separatrix surfaces.

Observing the evolution of magnetic field patches at high spatial resolution near the footpoints of magnetic loops may be crucial to understanding chromospheric and coronal heating. Recently, the Imaging Magnetograph eXperiment (IMaX) instrument on two flights of the SUNRISE balloon Mission (Solanki et al. 2010, 2017) has revealed glimpses of the photospheric magnetic field at much higher spatial resolution than before, namely, 0.15 arcsec, a factor of six better than the Helioseismic and Magnetic Imager (HMI) instrument on the Solar Dynamics Observatory (SDO; Pesnell et al. 2012). Using the observations from the first flight of SUNRISE, Smitha et al. (2017) tracked magnetic features with fluxes of $10^{15}-10^{18} \mathrm{Mx}$ in the Quiet Sun and found a flux emergence and cancellation rate of $1100 \mathrm{Mx} \mathrm{cm}^{-2} \mathrm{day}^{-1}$. This rate is an order of magnitude higher than previous measurements. Chitta et al. (2017b) observed the footpoints of extreme ultraviolet loops $(171 \AA)$ in a new active region. At $S D O / \mathrm{HMI}$ resolution $(1 \mathrm{arcsec})$ they appeared to be simple bipolar regions, with the loops joining two unipolar regions. However, higher-resolution maps at $0.15 \operatorname{arcsec}(100 \mathrm{~km})$ from IMaX on SUNRISE revealed mixed magnetic polarity at the loop footpoints, with flux cancellation at a rate of $10^{15} \mathrm{Mx} \mathrm{s}^{-1}$ (Figure 1).

It is well known that flux cancellation can liberate magnetic energy through reconnection. The general relevance of such flux events and associated reconnection for chromospheric and coronal energetics certainly needs further scrutiny. Indeed, three other pieces of evidence support the possible importance of flux cancellation for chromospheric and coronal heating. First, the heating of coronal loops may often be focused near their feet (e.g., Priest et al. 2000; Aschwanden 2008). Second, the well-established view that at least X-ray bright points are produced mainly by flux cancellation is supported by observations (e.g., Martin et al. 1985; Falconer et al. 1999) and theory (Priest et al. 1994; Parnell \& Priest 1995; Longcope 1998; Parnell \& Galsgaard 2004; Archontis \& Hansteen 2014). Third, the driving of magnetic reconnection by flux emergence or cancellation has different observational consequences depending on the location in height of the reconnection, which in turn depends on the magnitudes of the flux source and the overlying field strength (see Section 2). Thus, energy release can produce: an Ellerman bomb in the wings of $\mathrm{H} \alpha$ low down in the atmosphere around sunspots or in the Quiet Sun (Rouppe van der Voort et al. 2016; Hansteen et al. 2017); ultraviolet (UV) bursts in the chromosphere of an active region (Peter et al. 2014); explosive events in the transition region (Brueckner \& Bartoe 1983; Innes et al. 2011), blinkers (Harrison 1997); and X-ray bright points and X-ray jets in the corona (Shibata et al. 1992; Shimojo et al. 2007).

Recent studies further emphasize the possible, widespread role of reconnection during flux cancellation as the source of coronal loop brightenings. Tiwari et al. (2014) and Huang et al. (2018) discussed examples of flux cancellation triggering coronal brightening in apparently braided loops. Chitta et al. (2017a) observed that coronal loops in an evolved active region respond to an underlying ultraviolet burst and bidirectional jets, which in turn are triggered by magnetic reconnection at heights of $500 \mathrm{~km}$ above the photosphere driven by magnetic interactions leading to flux cancellation (Tiwari et al. 2017). Furthermore, Chitta et al. (2018) observed flux cancellation near the footpoints of coronal loops hosting nanoflares in the core of an active region. They identified complex mixed polarity field at the loop footpoints, where flux was canceling at a rate of $10^{15} \mathrm{Mx} \mathrm{s}^{-1}$. Plasma at $1 \mathrm{MK}$ in $171 \AA$ showed fluctuations at one footpoint where flux cancellation was occurring, and a steady evolution at the other footpoint. By comparing the energy content of the loop with that of the magnetic energy below the chromosphere (where reconnection is presumed to take place), they concluded that the analyzed flux cancellation events provide sufficient energy to heat the corona to temperatures exceeding $5 \mathrm{MK}$. 

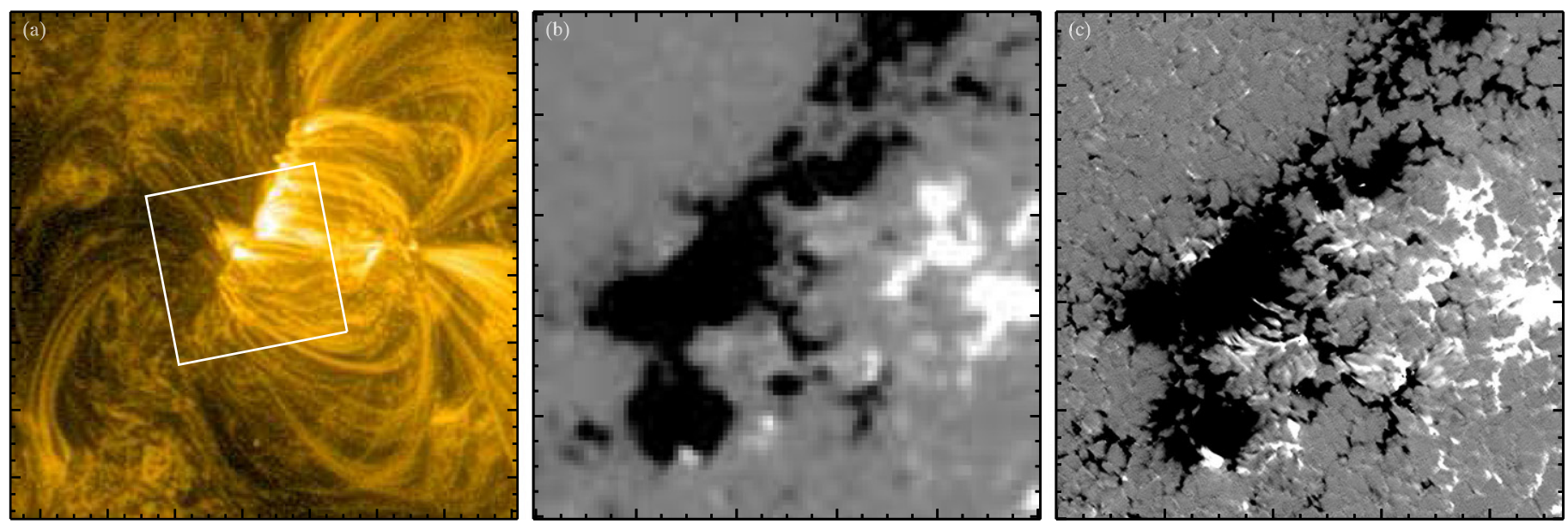

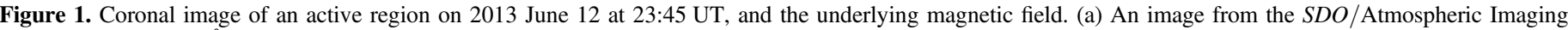

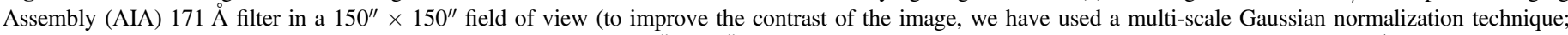

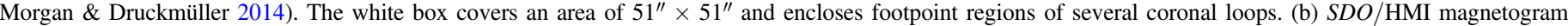

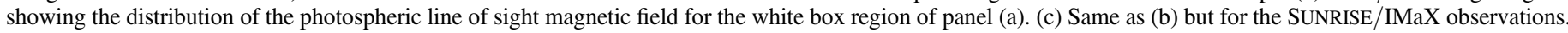
The magnetic flux density is saturated at $\pm 250 \mathrm{G}$. See Chitta et al. (2017b) for further details.

The realization that there is very much more photospheric flux cancellation than previously thought leads us to consider flux cancellation as a possible cause of chromospheric and coronal heating. We present some theoretical aspects (Section 2) and conclude with a discussion (Section 3), in which the height and amount of energy release are estimated as functions of the flux and overlying field strength.

\section{Theory for Energy Release at a Reconnecting Current Sheet}

Here we make some theoretical estimates of the energy release by steady-state magnetic reconnection in three dimensions, using basic theory (Priest 2014) and developing it in new ways. We calculate the rate of magnetic energy conversion when flux cancellation drives reconnection as two oppositely directed photospheric magnetic sources approach and cancel in an overlying field that is for simplicity here assumed horizontal (Orozco Suárez \& Bellot Rubio 2012; Stenflo 2013). Inclined fields will be treated in the future.

\subsection{Basic Properties of the Configuration}

Suppose that a photospheric source of negative parasite polarity of flux $-F_{p}$ lies next to a larger source $\left(F \geqslant-F_{p}\right)$ of positive polarity. The sources are at points $\mathrm{A}(-d \cos \alpha,-d \sin \alpha)$ and $\mathrm{B}$ $(d \cos \alpha, d \sin \alpha)$, a distance $2 d$ apart in the $x y$-plane and inclined at an angle $\alpha$ to the direction of an overlying field of strength $B_{0}$. Consider what happens as they approach one another at speeds $\pm v_{0}$ along the line joining $\mathrm{A}$ to $\mathrm{B}$.

The magnetic field above the photosphere $(y>0)$ is

$$
\boldsymbol{B}=\frac{F \hat{\boldsymbol{r}}_{1}}{2 \pi r_{1}^{2}}-\frac{F_{p} \hat{\boldsymbol{r}}_{2}}{2 \pi r_{2}^{2}}+B_{0} \hat{\boldsymbol{x}},
$$

where

$$
\begin{aligned}
& \boldsymbol{r}_{1}=(x-d \cos \alpha) \hat{\boldsymbol{x}}+(y-d \sin \alpha) \hat{\boldsymbol{y}}+z \hat{z}, \\
& \boldsymbol{r}_{2}=(x+d \cos \alpha) \hat{\boldsymbol{x}}+(y+d \sin \alpha) \hat{\boldsymbol{y}}+z \hat{\boldsymbol{z}},
\end{aligned}
$$

are the vector distances from the two sources to a point $\mathrm{P}(x$, $y, z)$.
Figure 2 sketches the evolution of the topology of the magnetic field in the horizontal $x y$-plane and the vertical $x z$ plane. Consider what happens when the distance $(2 d)$ between the two sources decreases from a large value. When the sources are so far apart that $d>d_{c}$, say, then there are two separatrix surfaces (containing two null points $\mathrm{N}_{1}$ and $\mathrm{N}_{2}$ ) that completely surround the fluxes that enter $\mathrm{A}$ and leave $\mathrm{B}$, so that no flux links A to B (Figure 2(a)). On the other hand, when $d=d_{c}$, a separator bifurcation occurs in which these two separatrices touch at a separator field line (S) that lies in the photospheric plane $(z=0)$ and joins the two null points (Figure 2(b)). Furthermore, when $d<d_{c}$, the separator rises above $z=0$ and a new domain is created bounding magnetic flux that passes under S and links source A to source B (Figure 2(c)). Finally, when $d=0$, the parasitic flux has completely canceled, leaving a separatrix surface that encloses the flux from the remaining dominant polarity (Figure 2(d)).

Here we focus on the particular case of equal flux sources $\left(-F_{p}=F\right)$ with $\alpha=0$ (Figure 3), so that the line joining the flux sources is aligned with the overlying magnetic field and we can take the analysis much further while retaining the main physics.

\subsection{Equal Flux Sources Aligned with Overlying Field $\left(-F_{p}=F, \alpha=0\right)$}

Consider in detail what happens as the flux sources approach one another as $d$ decreases. A natural length-scale for the configuration is the interaction distance (Longcope 1998)

$$
d_{0}=\left(\frac{F}{\pi B_{0}}\right)^{1 / 2} \text {. }
$$

Consider in detail what happens as the flux sources approach one another. When $d>d_{0}$, there is no flux connecting the sources (Figure 3(a)) and two first-order null points lie on the $x$-axis between the sources. When $d=d_{0}$, there is a local bifurcation in which the nulls combine to give a high-order null at the origin (Figure 3(b)). When $d<d_{0}$, a new semicircular separator is born in the $y z$-plane and its intersection with the $x z$ plane (marked S in Figure 3(c)) rises along the $z$-axis to height $z_{\mathrm{S}}$, say, so that magnetic flux now lies under the separator and 

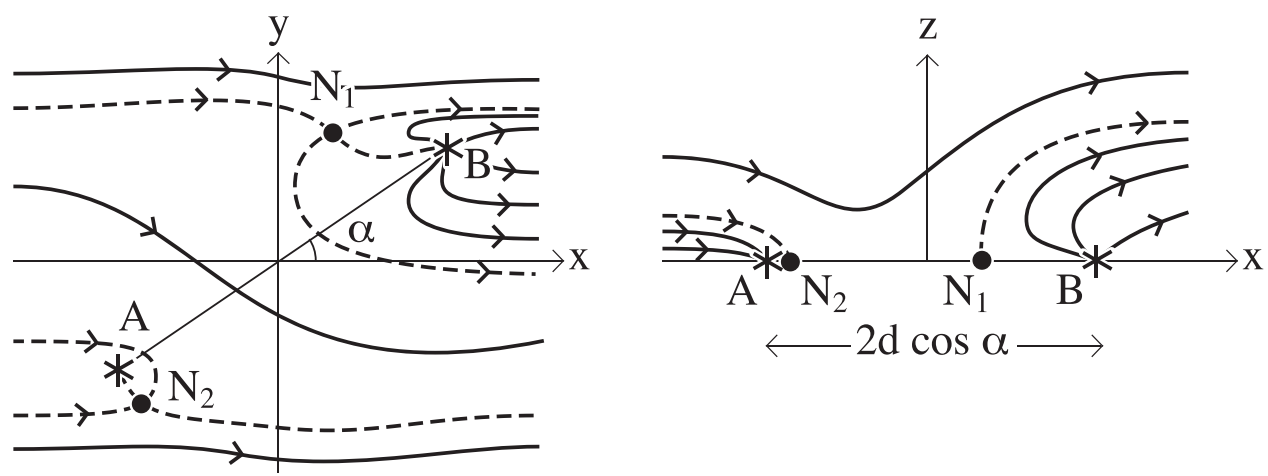

(a) $d>d_{c}$
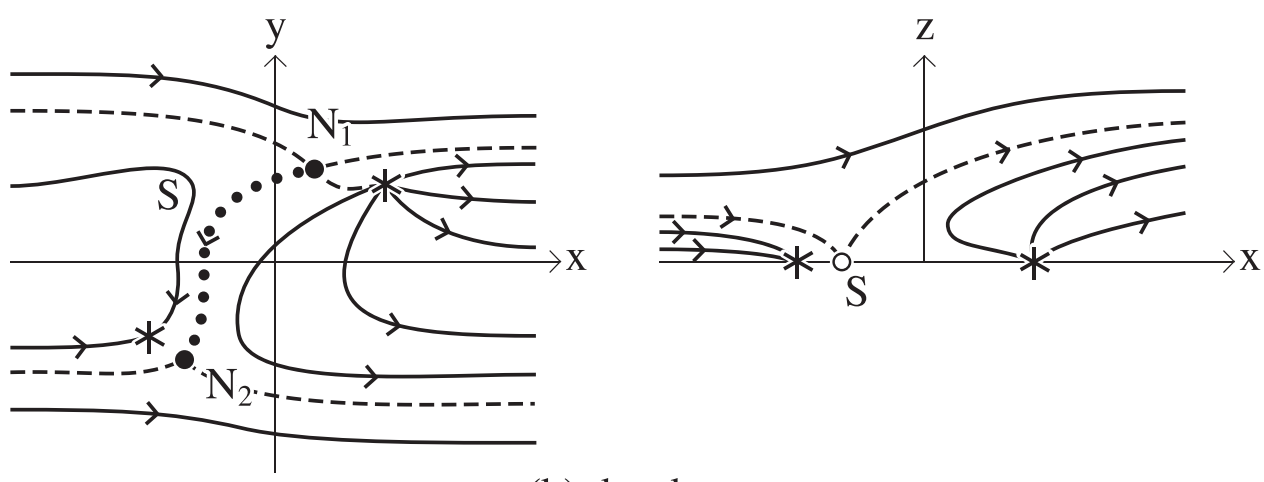

(b) $d=d_{c}$
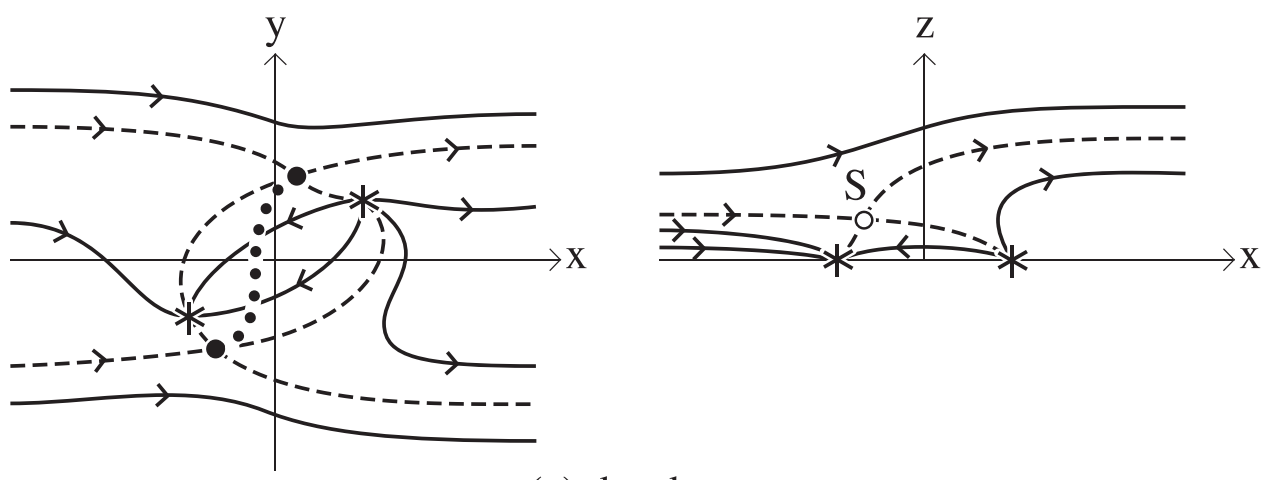

(c) $\mathrm{d}<\mathrm{d}_{\mathrm{c}}$
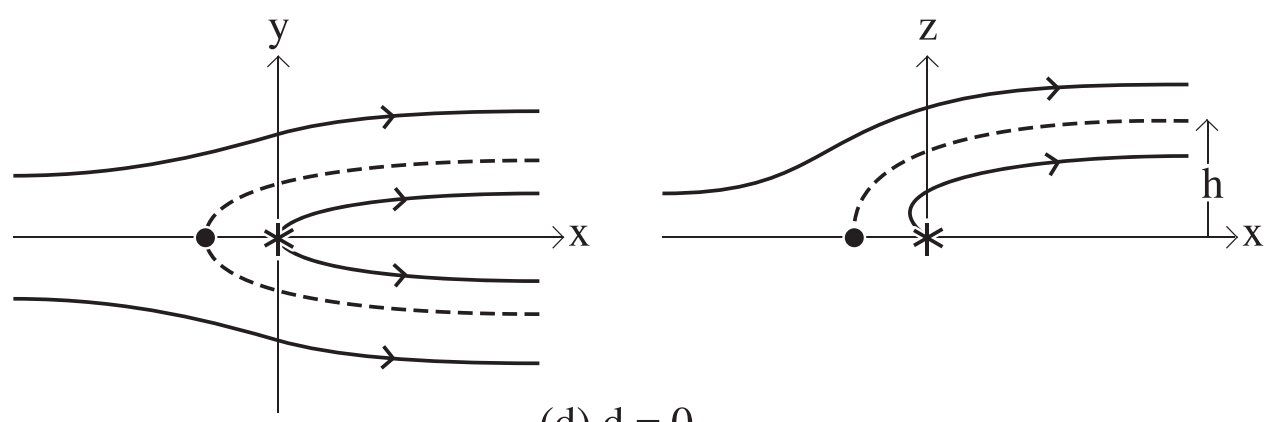

(d) $d=0$

Figure 2. Magnetic field structure seen from above (left) and from the side (right) during the approach along the line AB of oppositely directed photospheric sources (stars) of flux $-F_{p}$ and $F\left(\geqslant-F_{p}\right)$, separated by a distance $2 d$ and situated in an overlying uniform horizontal magnetic field $\left(B_{0}\right)$. Separatrix magnetic field lines are dashed, other magnetic field lines are solid curves, null points are large solid dots, and a separator ( $\mathrm{S}$ ) is a curve of dots seen from above and an unfilled dot when seen from the side. (a) is for $d>d_{c}$, (b) is for $d=d_{c}$, when a separator first appears lying in $z=0$, (c) is for $d<d_{c}$ when the separator arches above the surface, and (d) is for $d=0$.

connects the sources. The magnetic field is axisymmetric about the $x$-axis and so the separator is actually a semicircular ring of null points at distance $z_{\mathrm{S}}$ from the origin in every plane through the $x$-axis. In the case of unequal flux sources, the separator becomes a field line joining two nulls that lie in the $x y$-plane, as shown in Figure 2(c). 


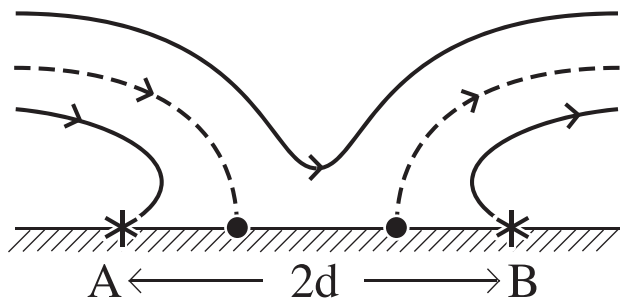

(a) $d>d_{o}$

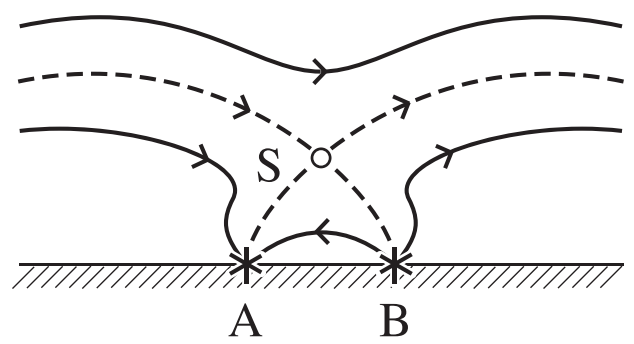

(c) $\mathrm{d}<\mathrm{d}_{\mathrm{o}}$

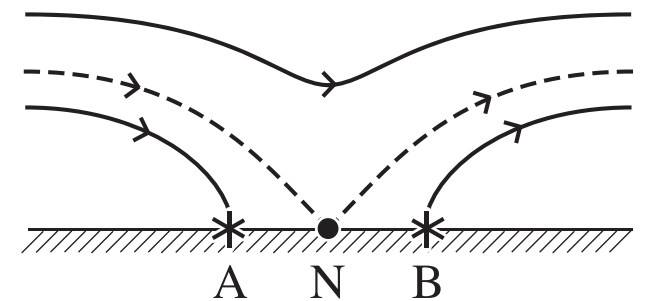

(b) $d=d_{o}$

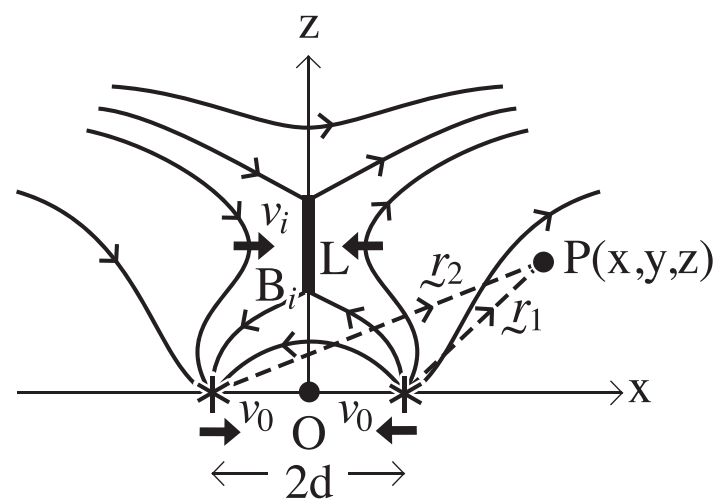

(d) $d=0$

Figure 3. Magnetic topologies in the vertical $x z$-plane when $\alpha=0$ and $-F_{p}=F$ for (a) $d>d_{0}$, (b) $d=d_{0}$ and (c) $d<d_{0}$ in terms of the interaction distance $\left(d_{0}=\left[F /\left(\pi B_{0}\right)\right]^{1 / 2}\right)$. (d) shows the notation when there is a reconnection region of length $L$ at which the inflow velocity and magnetic field are $v_{i}$ and $B_{i}$.

Along the $z$-axis, $B_{y}=B_{z}=0$ and

$$
\frac{B_{x}}{B_{0}}=-\frac{d d_{0}^{2}}{\left(d^{2}+z^{2}\right)^{3 / 2}}+1
$$

The location $\left(z=z_{\mathrm{S}}\right)$ of the null where the field vanishes is therefore given by

$$
z_{\mathrm{S}}^{2}=d^{2 / 3} d_{0}^{4 / 3}-d^{2}
$$

and is sketched in Figure 4(a) as a function of $d$. When $d=d_{0}$, the null is located at the origin, and, as $d$ decreases it rises along the $z$-axis to a maximum of $\left(z_{\mathrm{s}}\right)_{\max }=(4 / 27)^{1 / 4} d_{0}$ at $d=(1 / 3)^{3 / 4} d_{0}$. Thereafter, the null falls back to the origin as $d \rightarrow 0$.

The maximum height of the null point varies with $B_{0}$ and $F$, as shown in Figure 4(b). The height is typically about $0.6 d_{0}$, and so it lies in the chromosphere when $F$ is small enough or $B_{0}$ large enough. As the flux sources approach, the null point rises from the photosphere to its maximum height and then falls, but the energy that is released may spread to larger heights along the separatrix field lines that link to the reconnection site.

Note that in the more general case where the magnitudes of the two fluxes are not equal, when all the parasitic polarity flux has canceled, we are left with the situation shown in Figure 2(d). Here the flux from the remaining major polarity reaches a maximum height $h$, say, to which the field line from the null point asymptotes. It may be estimated from the equation of the field line in the plane $y=0$ through the null $(-1 / \sqrt{2}, 0,0)$, namely,

$$
\frac{1}{2} z^{2}-\frac{\frac{1}{2} x d_{0}^{2}}{\left(z^{2}+x^{2}\right)^{1 / 2}}=\frac{1}{2} d_{0}^{2} .
$$

Thus, as $x \rightarrow \infty$ on that field line, $z \rightarrow h$ and we find $h=\sqrt{2} d_{0}$.

\subsection{The Input Plasma Speed $\left(v_{i}\right)$ and Magnetic Field $\left(B_{i}\right)$ at the Reconnection Region}

When analyzing flux cancellation, the natural parameters for each value of the source separation $(2 d)$ are the interaction distance $\left(d_{0}\right)$, the flux source speed $\left(v_{0} \equiv \dot{d} \equiv d d / d t\right)$, and the overlying field strength $\left(B_{0}\right)$. We now therefore proceed to calculate the inflow speed $\left(v_{i}\right)$ and magnetic field $\left(B_{i}\right)$ to the current sheet and the sheet length $(L)$ as functions of those parameters for fast reconnection. The corresponding magnetic configuration for the reconnection is shown in Figure 3(d).

First of all, consider $B_{i}$. If the potential field near a null point has the form $B_{x}=k z$, then, when a current sheet forms, the magnetic field at the inflow to the sheet becomes $B_{i}=\frac{1}{2} k L$. Thus, after using Equation (4) to find $k$, we obtain

$$
\frac{B_{i}}{B_{0}}=\frac{3\left[1-\left(d / d_{0}\right)^{4 / 3}\right]^{1 / 2}}{2\left(d / d_{0}\right)^{1 / 3}} \frac{L}{d_{0}} .
$$

Next, consider $v_{i}$, which may be calculated from the rate of change $(\dot{\psi} \equiv d \psi / d t)$ of magnetic flux through the surface bounded by the $y$-axis and a semicircle of radius $z_{\mathrm{S}}$ out of the plane of Figure 3(c). After using Faraday's Law and $\boldsymbol{E}+\boldsymbol{v} \times \boldsymbol{B}=0$, this rate of change of flux becomes

$$
\dot{\psi} \equiv \frac{d \psi}{d t}=-\pi z_{\mathrm{S}} E=\pi z_{\mathrm{S}} v_{i} B_{i}
$$


(a)

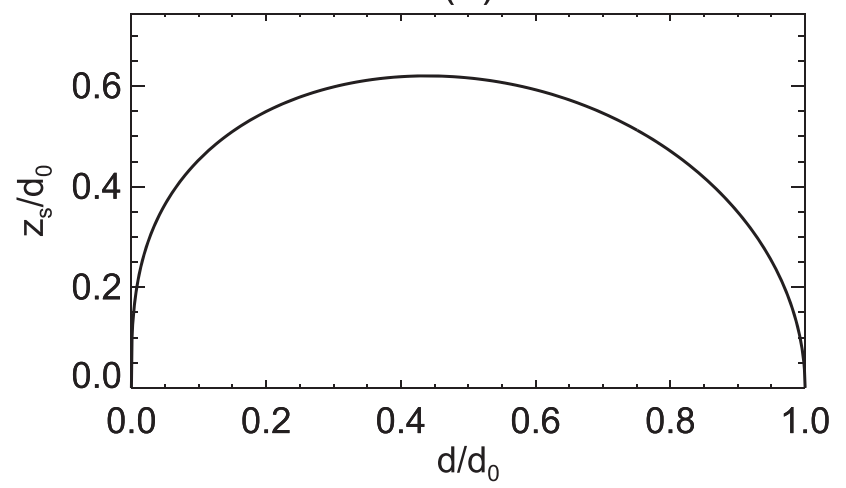

(b)

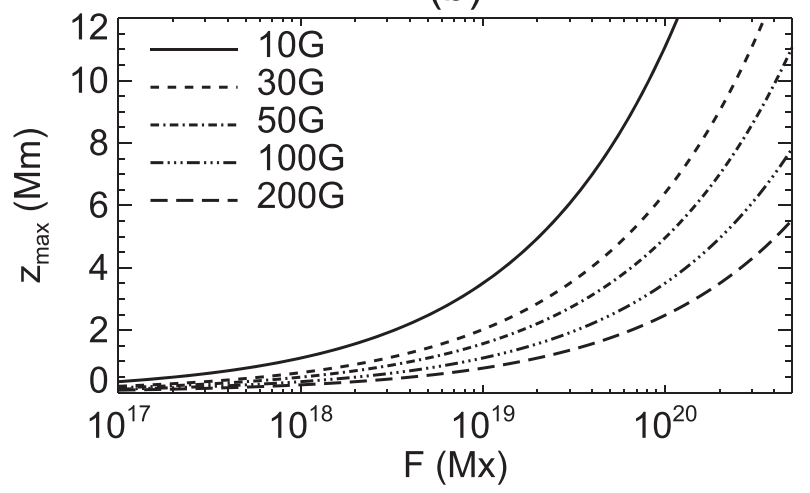

(c)

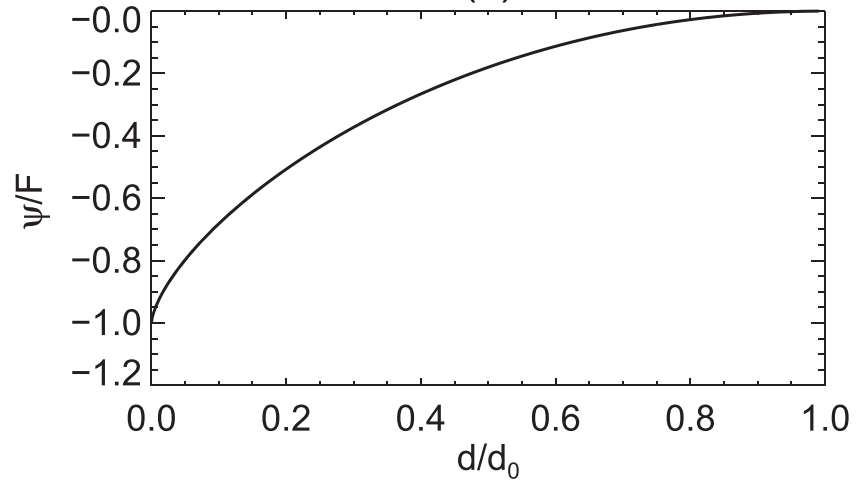

Figure 4. (a) The height $\left(z_{\mathrm{s}}\right)$ of the null point in Figure 3(c) as a function of the half-separation $(d)$ between the two sources, where $d_{0}=\left[F /\left(\pi B_{0}\right)\right]^{1 / 2}$ is the interaction distance. (b) The maximum height of the null point as a function of the flux $F$ for different values of the overlying magnetic field $B_{0}$. (c) The flux $(\psi)$ below the null point as a function of $d$.

However, $\psi$ may be calculated from the magnetic flux below $z_{\mathrm{S}}$ through the surface, namely,

$$
\psi=\int_{0}^{z_{\mathrm{S}}} \pi z B_{x} d z=F\left[\frac{3}{2}\left(\frac{d}{d_{0}}\right)^{2 / 3}-\frac{1}{2}\left(\frac{d}{d_{0}}\right)^{2}-1\right]
$$

This is sketched in Figure 4(c), from which it can be seen that, as expected, the reconnected flux vanishes when $d=d_{0}$ and increases monotonically in magnitude to $F$ as the separation (2d) between the sources approaches zero.

After differentiating $\psi$ with respect to $t$, we find

$$
\dot{\psi}=\frac{v_{0} F}{d_{0}}\left[\left(\frac{d}{d_{0}}\right)^{-1 / 3}-\frac{d}{d_{0}}\right] .
$$

Then, after substituting into Equation (7) for $\dot{\psi}$ from this equation, for $z_{\mathrm{S}}$ from Equation (5), and for $B_{i}$ from Equation (6), the required expression for $v_{i}$ becomes

$$
v_{i}=\frac{2 v_{0}}{3} \frac{d_{0}}{L}\left(\frac{d_{0}}{d}\right)^{1 / 3} \text {. }
$$

\subsection{Energy Release by Fast Reconnection}

Three possibilities have been studied for fast reconnection, all of which may occur within our model, depending on the microscopic plasma physics at work. First, according to Petschek or Almost-Uniform reconnection theory (e.g., Priest 2014), the internal structure of the reconnection region consists of a central small sheet and four slow-mode shock waves, at which most of the energy conversion takes place, with 0.4 of the inflowing magnetic energy being converted to heat. Second, collisionless reconnection is aided by the Hall effect, when the resistive diffusion region is replaced by an ion diffusion region and a smaller electron diffusion region, but the same fast maximum rate of reconnection results (e.g., Shay \& Drake 1998; Birn \& Priest 2007). Third, when the central sheet is long enough, it goes unstable to secondary tearing mode instability and a regime of impulsive bursty reconnection results (e.g., Priest 1986; Loureiro et al. 2007), with a mean energy conversion and reconnection rate similar to the other cases.

The rate of inflow of magnetic energy from one side at speed $v_{i}$, and with field $B_{i}$ and density $\rho_{i}$ through a surface with height $L$ and extending a distance $\pi z_{\mathrm{S}}$ along the current sheet at the separator, is just the Poynting influx $\left(E H_{i} L \pi z_{\mathrm{S}}=E B_{i} L \pi z_{\mathrm{S}} / \mu\right)$. However, the magnitude of the electric field is $E=v_{i} B_{i}$, and an equal amount of magnetic energy flows in from the other side of the sheet, so that the total rate of conversion of energy to heat from both sides is

$$
\frac{d W}{d t}=0.8 \frac{v_{i} B_{i}^{2}}{\mu} L \pi z_{\mathrm{s}}
$$

$L$ is determined by the condition that the inflow speed $v_{i}=\alpha v_{A i}$, where $\alpha$ is typically between 0.01 and 0.1 , and $v_{A i}=B_{i} / \sqrt{\mu \rho_{i}}$ is the inflow Alfvén speed. Then, after setting $\alpha v_{A i}=\alpha\left(B_{i} / B_{0}\right) v_{A 0}$ in Equation (9), where $v_{A 0}=B_{0} / \sqrt{\mu \rho_{i}}$ is a hybrid Alfvén speed, and using Equation (6), we obtain

$$
\frac{L^{2}}{d_{0}^{2}}=\frac{4 v_{0}}{9 \alpha v_{A 0}} \frac{1}{\left[1-\left(d / d_{0}\right)^{4 / 3}\right]^{1 / 2}} .
$$

After substituting for $B_{i} / B_{0}$ from Equation (6), $v_{i} / v_{A 0}$ from Equation (9), and $L$ from Equation (11), the rate of energy conversion Equation (10) becomes

$$
\frac{d W}{d t}=0.8 \frac{2 \pi}{3} \frac{v_{0} B_{0}^{2}}{\mu} d_{0}^{2} \frac{M_{A 0}}{\alpha} \frac{\left[1-\left(d / d_{0}\right)^{4 / 3}\right]}{\left(d / d_{0}\right)^{2 / 3}}
$$

for a given flux source speed $v_{0}$, overlying field $B_{0}$, interaction distance $d_{0}$, Alfvén Mach number $\left(M_{A 0}=v_{0} / v_{A 0}\right)$, and source separation $2 d$.

\section{Discussion}

Inspired by the remarkable SUNRISE observations, we here propose that magnetic reconnection driven by photospheric flux cancellation may be a ubiquitous mechanism for powering coronal loops and also for releasing heat in the chromosphere. 
We suggest the outlines of a theoretical model for the interaction between two opposite-polarity sources of flux $\pm F$ in an overlying horizontal field $B_{0}$, which can be greatly developed in future by sophisticated computational experiments.

Three key roles are played by the interaction distance, which may be written as

$$
d_{0}=6\left(\frac{F_{19}}{B_{1}}\right)^{1 / 2} \mathrm{Mm},
$$

where $F_{19}$ is the flux in units of $10^{19} \mathrm{Mx}$, and $B_{1}$ is the overlying field in units of $10 \mathrm{G}$. The first is that, as the opposite polarity sources approach one another, they drive reconnection as soon as $d<d_{0}$. For example, small flux sources of $10^{17} \mathrm{Mx}$ give values for $d_{0}$ of $0.6 \mathrm{Mm}$ in a $10 \mathrm{G}$ field or $0.2 \mathrm{Mm}$ in a $100 \mathrm{G}$ field. On the other hand, large flux sources of $10^{20} \mathrm{Mx}$, give values of $19 \mathrm{Mm}$ in a $10 \mathrm{G}$ field and $6 \mathrm{Mm}$ in a $100 \mathrm{G}$ field.

The second role is to determine the maximum height $\left(\left(z_{\mathrm{s}}\right)_{\max } \approx 0.6 d_{0}\right)$ for the reconnection location and so explain why flux cancellation sometimes leads to energy release in the photosphere, sometimes in the chromosphere and sometimes in the transition region or corona. Thus, $\left(z_{\mathrm{S}}\right)_{\max }$ lies in the photosphere if $F<2 \times 10^{17} \mathrm{Mx}$ for $B_{0}=10 \mathrm{G}$, or $F<2 \times 10^{18} \mathrm{Mx}$ for $B_{0}=100 \mathrm{G}$. On the other hand, it lies in the chromosphere if $2 \times 10^{17}<F<3 \times 10^{18} \mathrm{Mx}$ for $B_{0}=10 \mathrm{G}, \quad$ or $2 \times 10^{18}<F<3 \times 10^{19} \mathrm{Mx}$ for $B_{0}=$ $100 \mathrm{G}$. These computed maximum reconnection heights are consistent with those from magnetic field extrapolations for chromospheric bursts (e.g., Chitta et al. 2017a; Tian et al. 2018).

The third role for $d_{0}$ is that, when the overlying field is horizontal, the height reached by the field lines that link to the reconnection site varies between $1.4 d_{0}$ when reconnection starts and $1.5 d_{0}$ when the reconnection height peaks (at $\left.\left(z_{\mathrm{S}}\right)_{\max }\right)$ ). Thus, we expect energy to propagate down toward the photosphere and up to a height of 1.4-1.5 $d_{0}$. This lies purely within the photosphere and chromosphere when $F<4 \times 10^{17} \mathrm{Mx}$ for $B_{0}=10 \mathrm{G}$ or $F<4 \times 10^{18} \mathrm{Mx}$ for $B_{0}=100 \mathrm{G}$. Of course, the height will be much larger when the field lines are inclined to the solar surface.

Next, consider the energy liberated. In order to heat the Quiet-Sun chromosphere and corona, we need $4 \times 10^{6}$ and $3 \times 10^{5} \mathrm{erg} \mathrm{cm}^{-2} \mathrm{~s}^{-1}$, respectively, whereas in an active region the corresponding needs are $2 \times 10^{7}$ and $10^{7} \mathrm{erg} \mathrm{cm}^{-2} \mathrm{~s}^{-1}$, respectively. Let us evaluate the rate of heat produced in the chromosphere from Equation (12) with typical values of $d=0.4 d_{0}$ and $\alpha=0.1$ (Priest 2014). Then the expression (12) may be written as

$$
\frac{d W}{d t}=5 \times 10^{22} v_{4} B_{1} F_{18} M_{A 0} \mathrm{erg} \mathrm{s}^{-1},
$$

where $v_{4}$ is $v_{0}$ in units of $10^{4} \mathrm{~cm} \mathrm{~s}^{-1}$. Thus, for example, in the Quiet Sun, if we adopt values of $v_{0}=1 \mathrm{~km} \mathrm{~s}^{-1}, F=10^{18} \mathrm{Mx}$, $B_{0}=10 \mathrm{G}, M_{A 0}=0.1$ (Priest 2014), so that an area of $l_{0}^{2}$ is swept out in a time of, say, $10^{3} \mathrm{~s}$, where $l_{0}=10^{8} \mathrm{~cm}$, then the heating per unit area is

$$
\frac{1}{l_{0}^{2}} \frac{d W}{d t}=5 \times 10^{6} \mathrm{erg} \mathrm{cm}^{-2} \mathrm{~s}^{-1}
$$

which is sufficient to heat the Quiet-Sun chromosphere. On the other hand, a flux of $F=10^{19} \mathrm{Mx}$ and an overlying field of $B_{0}=100 \mathrm{G}$ with $M_{A 0}=0.01$, characteristic of active regions would give a corresponding value of $5 \times 10^{7} \mathrm{erg} \mathrm{cm}^{-2} \mathrm{~s}^{-1}$, which is sufficient for the active-region chromosphere. In turn, if $10 \%-20 \%$ of this leaks through to higher levels, it would be sufficient to heat the corona.

We have proposed a ubiquitous way of creating nanoflares near the base of chromospheric and coronal loops with sufficient energy to power the chromosphere and corona, building on previous flux cancellation theory (e.g., Parnell \& Priest 1995; Welsch 2006). In future, it will be interesting to develop the model further by means of computational experiments, in order to investigate the nature of the energy release and its propagation along magnetic loops from the reconnection source.

The authors are most grateful for invaluable discussions with Hardi Peter and Clare Parnell. L.P.C. received funding from the European Union's Horizon 2020 research and innovation programme under the Marie Skłodowska-Curie grant agreement No. 707837. The German contribution to SUNRISE and its reflight was funded by the Max Planck Foundation, the Strategic Innovations Fund of the President of the Max Planck Society (MPG), DLR, and private donations by supporting members of the Max Planck Society. The Spanish contribution was funded by the Ministerio de Economía y Competitividad under Projects ESP2013-47349-C6 and ESP2014-56169-C6, partially using European FEDER funds. The HAO contribution was partly funded through NASA grant number NNX13AE95G. SDO data are courtesy of NASA/SDO and the AIA, and HMI science teams.

\section{ORCID iDs}

E. R. Priest (1) https://orcid.org/0000-0003-3621-6690

L. P. Chitta (1) https://orcid.org/0000-0002-9270-6785

P. Syntelis $\odot$ https://orcid.org/0000-0002-6377-0243

\section{References}

Archontis, V., \& Hansteen, V. 2014, ApJL, 788, L2

Aschwanden, M. J. 2008, ApJL, 672, L135

Birn, J., \& Priest, E. R. 2007, Reconnection of Magnetic Fields: MHD and Collisionless Theory and Observations (Cambridge: Cambridge Univ. Press)

Brueckner, G., \& Bartoe, J.-D. F. 1983, ApJ, 272, 329

Chitta, L. P., Peter, H., \& Solanki, S. K. 2018, A\&A, 615, L9

Chitta, L. P., Peter, H., Solanki, S. K., et al. 2017b, ApJS, 229, 4

Chitta, L. P., Peter, H., Young, P. R., \& Huang, Y.-M. 2017a, A\&A, 605, A49

Falconer, D. A., Moore, R. L., Porter, J. G., \& Hathaway, D. H. 1999, SSRv, 87,181

Hansteen, V. H., Archontis, V., Pereira, T. M. D., et al. 2017, ApJ, 839, 22

Harrison, R. A. 1997, SoPh, 175, 467

Huang, Z., Mou, C., Fu, H., et al. 2018, ApJL, 853, L26

Innes, D. E., Cameron, R. H., \& Solanki, S. K. 2011, A\&A, 531, L13

Klimchuk, J. A. 2006, SoPh, 234, 41

Longcope, D. W. 1998, ApJ, 507, 433

Loureiro, N. F., Schekochihin, A. A., \& Cowley, S. C. 2007, PhPl, 14, 100703

Martin, S. F., Livi, S., \& Wang, J. 1985, AuJPh, 38, 929

Morgan, H., \& Druckmüller, M. 2014, SoPh, 289, 2945

Orozco Suárez, D., \& Bellot Rubio, L. R. 2012, ApJ, 751, 2

Parker, E. N. 1988, ApJ, 330, 474

Parnell, C. E., \& De Moortel, I. 2012, RSPTA, 370, 3217

Parnell, C. E., \& Galsgaard, K. 2004, A\&A, 428, 595

Parnell, C. E., \& Priest, E. R. 1995, GApFD, 80, 255

Pesnell, W. D., Thompson, B. J., \& Chamberlin, P. C. 2012, SoPh, 275, 3 
Peter, H., Tian, H., Curdt, W., et al. 2014, Sci, 346, 1255726

Priest, E. 1986, MitAG, 65, 41

Priest, E. R. 2014, Magnetohydrodynamics of the Sun (Cambridge: Cambridge Univ. Press)

Priest, E. R., Foley, C. R., Heyvaerts, J., et al. 2000, ApJ, 539, 1002

Priest, E. R., Heyvaerts, J., \& Title, A. 2002, ApJ, 576, 533

Priest, E. R., Parnell, C. E., \& Martin, S. F. 1994, ApJ, 427, 459

Rouppe van der Voort, L. H. M., Rutten, R. J., \& Vissers, G. J. M. 2016, A\&A, 592, A100

Shay, M. A., \& Drake, J. F. 1998, GeoRL, 25, 3759

Shibata, K., Ishido, Y., Acton, L. W., et al. 1992, PASJ, 44, L173
Shimojo, M., Narukage, N., Kano, R., et al. 2007, PASJ, 59, 745

Smitha, H. N., Anusha, L. S., Solanki, S. K., \& Riethmüller, T. L. 2017, ApJS, 229,17

Solanki, S. K., Barthol, P., Danilovic, S., et al. 2010, ApJL, 723, L127

Solanki, S. K., Riethmüller, T. L., Barthol, P., et al. 2017, ApJS, 229, 2

Stenflo, J. O. 2013, A\&ARv, 21, 66

Tian, H., Zhu, X., Peter, H., et al. 2018, ApJ, 854, 174

Tiwari, S. K., Alexander, C. E., Winebarger, A. R., \& Moore, R. L. 2014, ApJL, 795, L24

Tiwari, S. K., Thalmann, J. K., Panesar, N. K., et al. 2017, ApJL, 843, L20

Welsch, B. T. 2006, ApJ, 638, 1101 